\title{
Fast Abdominal Medical Image Registration Based on Modified SIFT
}

\author{
Wang Beilei ${ }^{1}$, Meng Lu², Xu Jie ${ }^{1}$, Shao Ye ${ }^{1}$
}

\begin{abstract}
SIFT is an excellent descriptor to features in medical images. However, the algorithm to achieve SIFT is tedious and takes a long time. To cope with this, we combined image integration and image region partition and proposed a modified SIFT descriptor capable of describing the global energy information of the image. We further proposed a spatial SIFT -oriented non-rigid registration model. Experimental results indicated that fast and accurate registration could be achieved with this method.
\end{abstract}

Key words: SIFT; non-rigid registration; feature description; medical image

\section{Introduction}

Image registration has always been the centre of research. Image registration techniques have been widely used in medical image analysis, remote sensing data analysis and computer visualization. Image registration can be used to trace the effects of radiation therapy, to study the individual difference of human brains and to aid in diagnosing neurological diseases. Image registration can also be used to segment MRI images to reconstruct the $3 \mathrm{D}$ human brain images.

Feature-based registration is achieved by extracting two or more features (points, lines or surfaces), describing the features with certain parameters and registering the corresponding parameters[1]. Feature extraction can be divided into three categories: contour-based method, parameter model-based method and grey-level-based method.

\footnotetext{
'Wang Beilei $(\bowtie)$

School of Software, Northeastern University,

Shenyang 110819, China

e-mail: wangbeilei happy@163.com

${ }^{2}$ Meng Lu

School of Information Science \& Engineering, Northeastern University,

Shenyang 110819, China

${ }^{1} \mathrm{Xu}$ Jie

School of Software, Northeastern University,

Shenyang 110819, China

${ }^{1}$ Shao Ye

School of Software, Northeastern University,

Shenyang 110819, China

This work was supported by the National Natural Science Foundation of China (Grant Nos. $61374178,61202085)$
} 
In grey-level-based method, a parameter reflecting the feature points is calculated[2]. It is a feature point detection method with rotation invariance, position invariance, scale invariance and illumination invariance.

SIFT-based methods have been applied to various problems such as image tagging [3], object classification [4], large-scale mobile visual search [5] and duplicated regions detection[6].

The core idea of SIFT lies in processing images in multi-scale space and using extremes in each scale as feature points[7]. The chosen points are further screened using correction principles and the feature points for registration are preserved. Feature extraction in SIFT includes: (1) finding extremes in multi-scale space; (2) locating the extremes and finding the corresponding key points and their positions and scales; (3) assigning direction parameters to each key point (use the principal direction of key point gradient in its neighbour as the characteristic direction to achieve scale and direction invariance for the descriptor); (4) generating key points descriptor.

\section{Modification of SIFT features}

SIFT feature point descriptor with 128 dimensions are generated with gradient of the key point in its neighbour. Therefore, when similar textures exist in images, descriptors with similar or even same SIFT eigenvectors are generated. This will affect the matching of SIFT feature points or even lead to mismatching. One feature point in the reference image is matched with a couple of feature points in the floating image or multiple reference points in the reference image are matched with one feature point in the floating image. To solve this issue, we modified the traditional SIFT method and proposed a new SIFT feature point descriptor capable of reflecting the global energy information of images. In this modified method, image integration and image region partition are combined with SIFT and spatial distribution descriptors of feature points are calculated. SIFT feature point descriptor is weighed with spatial distribution descriptor to build a new feature point descriptor. Mismatch is corrected in the final step.

\section{1 image integration}

For a grey image, the value of its integral image at a certain point $(x, y)$ is the sum of pixels above and to the left of $(x, y)$ :

$$
\mathrm{I}(\mathrm{x}, \mathrm{y})=\operatorname{sum}(\mathrm{G}(\mathrm{I}, \mathrm{j}))
$$

where $0<=\mathrm{I}<=\mathrm{x}, 0<=\mathrm{j}<=\mathrm{y}$. I is the integral image and $\mathrm{G}$ is the grey image. According to its definition, the following is straight ford:

$$
I(x, y)=I(x-1, y)+I(y-1, x)-I(x-1, y-1)+G(i, j)
$$


Feature points considered here are mainly the edge points or points with large gradients. Thus, the concept of integral image can be slightly modified and consider only the edge points in above and to the left of $(x, y)$. Before integrating images, edge processing is conducted for the images (Canny operator is used in this case). Number of edge points in any rectangular can be obtained from integral image of the edge image. The number of edge points in abcs in Fig. 1.1 can be calculated using:

Total number $=\mathrm{d}+\mathrm{a}-\mathrm{c}-\mathrm{d}$

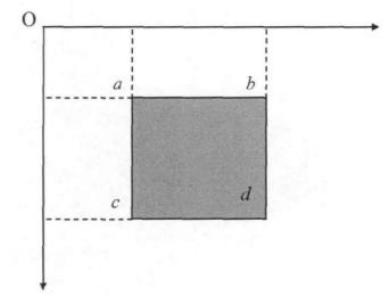

Fig. 1.1 Schematic diagram of integral image

\subsection{Image region partition}

Square with different sizes are generated with the SIFT feature points as their centres as shown in Fig. 1.2. One of the SIFT feature points is set as the origin of coordinate system. Starting from the second quadrant, square regions are generated anticlockwise with the side length varying according to its distance from the centre. Suppose that the square next to the centre point has a side length of $\mathrm{L}$. The side length increases by a multiple of $2^{\wedge} \mathrm{n}(\mathrm{n}=0,1,2,3,4 \ldots)$ until image edge is reached. Different squares have different effects on the SIFT feature point at the centre. The further away from the centre, the smaller effects the square has.

Square has been chosen as the partition shape to match with the integral image shape. After partitioning the image based on SIFT feature points, total number of edge points in each square region can be calculated according to Eq. 3 .

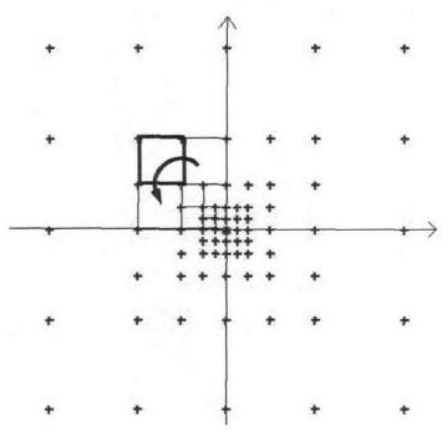

Fig. 1.2 Image region partition based on feature points 


\subsection{Image spatial distribution descriptor}

Image spatial distribution descriptor can be obtained after partitioning the image with SIFT feature points and calculating the total number of feature points in each region. The value of each component in the descriptor is the number of edge points in each square. The edge points are counted inside out following an anticlockwise order with the SIFT feature as the centre. As shown in Fig. 1.3, counting starts from the upper left square and goes further to the lower left, lower right and upper right square.

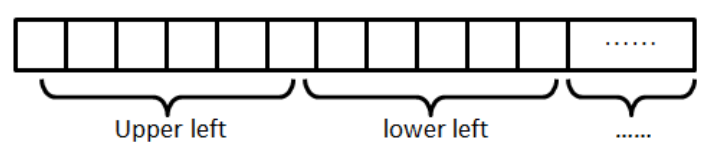

Fig. 1.3 Image spatial distribution descriptor

The image spatial distribution descriptor thus generated reflects the contour point distribution of the image whose centre is the SIFT feature point. It covers a much broader range than the traditional feature point-centred SIFT. Even for images with large similarity, feature points can still be distinguished.

Define the feature point descriptor of image I as $\mathrm{S}$ and spatial distribution descriptor as G. The spatial SIFT feature descriptor can be defined as the weighted combination of $\mathrm{S}$ and $\mathrm{G}$ in Eq. 4:

$$
F=\left[\begin{array}{l}
\omega S \\
(1-\omega) G
\end{array}\right]
$$

where $\omega$ is coefficient of weight.

\section{4 non-rigid registration model based on modified spatial SIFT features}

Optical flow filed is used to process image sequences and finds wide-spread application in medical image registration. It is based on the hypothesis that two consequent images are taken as extremely short time interval and corresponding pixels in both images have nearly constant grey level:

$$
I(x, y, t)=I(x+\Delta x, y+\Delta y, t+\Delta t)
$$

Expanding Eq. 5 according to Taylor's formula yields:

$$
\frac{\partial I}{\partial x} \cdot u+\frac{\partial I}{\partial y} \cdot v+\frac{\partial I}{\partial z}=0
$$

$$
\text { with } u=\frac{d x}{d t}, v=\frac{d y}{d t} \text {. }
$$


Vector field $(\mathrm{u}, \mathrm{v})$ is called the optical flow field. Image registration based on optical flow filed utilizes the optical flow filed between reference image $\mathrm{R}$ and floating image F. Transforming the floating image $F$ according to the optical flow filed yields image $\mathrm{R}$ in Eq. 7:

$$
F(x+u, y+v)=R(x, y)
$$

Traditional optical flow field-based registration model is modified in this section. Spatial SIFT feature points sets of the floating and reference images are registered using non-rigid feature constraint-based registration model:

$$
E(u, v)=E_{\text {data }}(u, v, t)+E_{\text {point }}(u, v)+E_{\text {smooth }}(u, v, t)
$$

where $\mathrm{E}_{\text {data }}$ is the data set, $\mathrm{E}_{\text {point }}$ is the regular term and $\mathrm{E}_{\text {smooth }}$ is the feature constrain.

$$
\begin{aligned}
& E_{\text {data }}=\sum_{k=1}^{3} \int_{\Omega} \psi\left(a b s\left(I_{1}(x, y, t)-I_{2}(x+u, y+v, t+1)\right) d x d y\right. \\
& E_{p o \text { int }}=\int_{\Omega} \delta(x, y) \omega(u, v) \psi\left(\left\|\omega(u, v)-\omega\left(u_{p o \text { int }}, v_{p o \text { int }}\right)\right\|^{2}\right) \\
& \delta(x, y)= \begin{cases}1 & x \in Q \\
0 & \text { else }\end{cases} \\
& \left.E_{\text {smooth }}=\int_{\Omega}\left(\alpha_{1}+\alpha_{2} b(x, y, t)\right) \psi\left(\|\nabla u\|^{2}+\|\nabla v\|^{2}\right)\right) d x d y \\
& b(x, y, t)=N\left(\left\|\nabla I_{1}\right\| ; \delta^{2}\right)
\end{aligned}
$$

\section{Results and discussion}

Clinical abdominal CT slices are used to test this method. The data set is in DICOM format with a resolution of $512 * 512 * 48$. The pixel size is $0.80 \mathrm{~mm}$ with a interlayer distance of $5 \mathrm{~m}$.

\subsection{Test on SIFT feature based registration}

The registration results are shown in Fig. 3.1. The total computation time was 258 ms. 606 and 686 SIFT feature points were extracted from the reference image and floating image respectively. Among them, 265 SIFT feature points were matched. The matching rate was $56.5 \%$. 


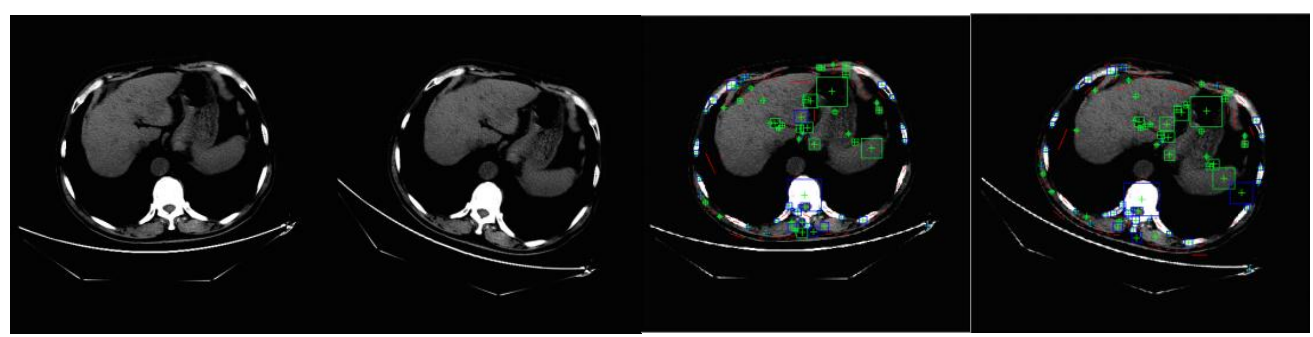

(a)

(b)

(c)

(d)

Fig. 3.1 SIFT feature points extraction and registration results

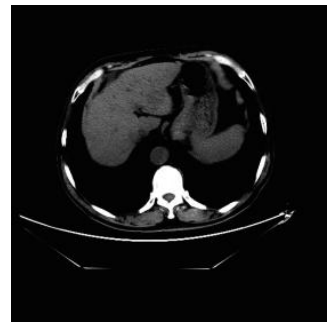

(a) reference image

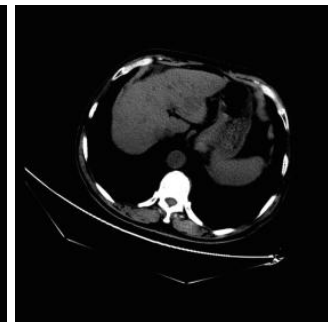

(b) floating image

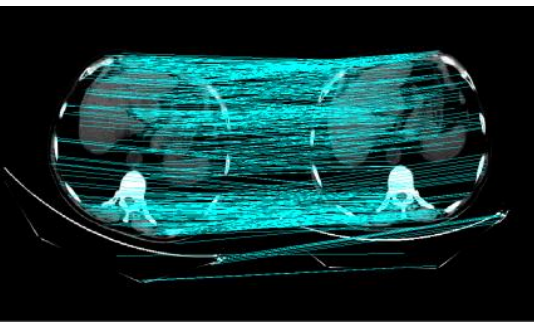

(c) SIFT feature points extraction and matching
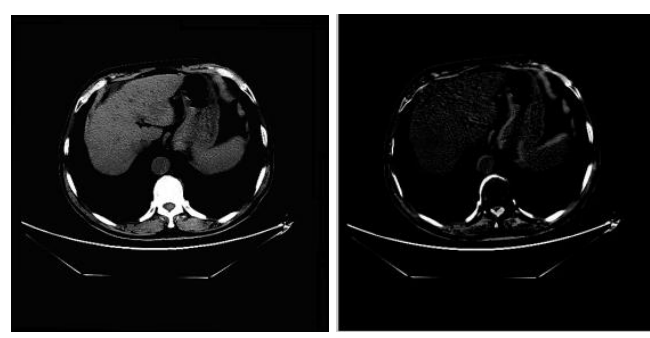

(d) after registration (e) difference image between floating and reference images

Fig. 3.2 SIFT feature point extraction and registration result

\subsection{Accelerated SIFT feature extraction}

The results obtained with traditional SIFT algorithm and the modified SIFT algorithm were compared. Images with resolution of $300 \times 200,640 \times 480,800 \times 640,1200 \times 800$ and $1600 \times 1200$ were used. The data were from BSDS500 data set of Computer Vision Group in UC Berkeley. A comparison of the algorithm proposed in this paper and the traditional CPU-based algorithm is shown in Table 1.

Table 1. Comparison of the modified algorithm and traditional CPU-based algorithm

\begin{tabular}{cccccc}
\hline Item & Image A & Image B & Image C & Image D & Image E \\
\hline Resolution & $300 \times 200$ & $640 \times 480$ & $800 \times 640$ & $1200 \times 800$ & $1600 \times 1200$ \\
$\begin{array}{c}\text { Number of SIFT feature points in CPU } \\
\text { architecture }\end{array}$ & 127 & 1746 & 3186 & 5761 & 8672 \\
$\begin{array}{c}\text { Computing time in CPU architecture } \\
\quad(\mathrm{ms})\end{array}$ & 365 & 1609 & 2815 & 5260 & 9053 \\
$\begin{array}{c}\text { Number of SIFT feature points in } \\
\text { CUDA architecture }\end{array}$ & 125 & 1781 & 3175 & 5736 & 8677 \\
$\begin{array}{c}\text { Computing time in CUDA architecture } \\
(\text { ms) }\end{array}$ & 135 & 311 & 361 & 426 & 463 \\
$\quad$ Acceleration ratio & 2.70 & 5.16 & 7.78 & 12.36 & 19.54 \\
\hline
\end{tabular}


In the test, parameters are set as follows: set number is determined by the image resolution; DOG has 3 layers in each set; the threshold for DOG function is 0.04; main curvature threshold is 10; feature descriptor has a seeded region size of 4 by 4 ; feature descriptor is projected in 8 directions within ite neighbour; Gaussian smoothing number $\sigma$ is 1.5 times the scale of the feature point in the principal direction.

Fig.3.3 indicates the correlation between feature point number and acceleration ratio.

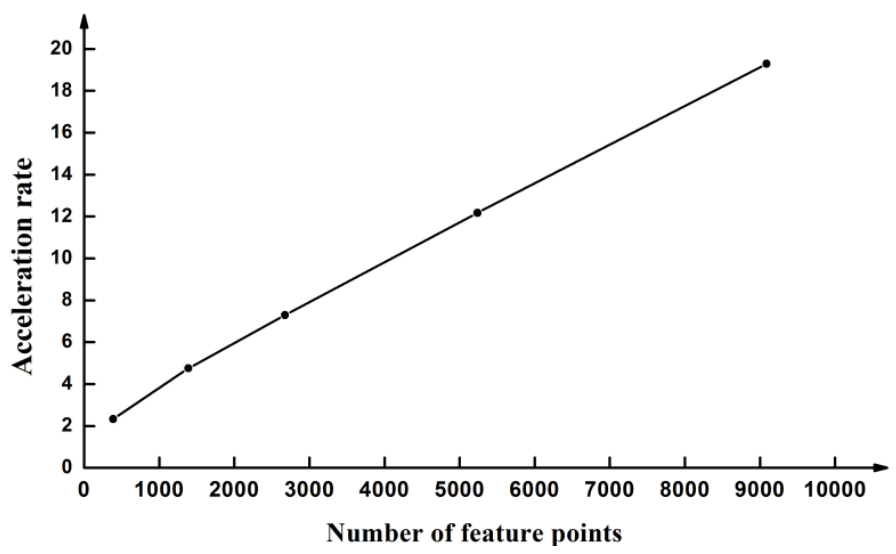

Fig. 3.3 Correlation between SIFT feature point number and acceleration ratio

\section{Conclusions}

SIFT is an excellent descriptor of features in medical images. It is one of the optimal options for feature-based image registration. Its intrinsic complexity and long computation time strongly limits its usage. This paper modified the tradition SIFT and proposed a new spatial SIFT based non-rigid registration model. Therefore, accurate and fast medical image registration can be achieved with this method.

\section{Acknowledgements}

This research was supported by the National Natural Science Foundation of China (Grant Nos. 61374178,61202085).

\section{Reference:}

1. Liao, K., G. Liu, and Y. Hui, An improvement to the SIFT descriptor for image representation and matching. Pattern Recognition Letters, 2013. 34(11): p. 12111220.

2. Cai, G.-R., et al., Perspective-SIFT: An efficient tool for low-altitude remote sensing image registration. Signal Processing, 2013. 93(11): p. 3088-3110.

3. Zhang, X., et al., Social image tagging using graph-based reinforcement on multitype interrelated objects. Signal Processing, 2013. 93(8): p. 2178-2189. 
4. Zhang, L., et al., Fast multi-view segment graph kernel for object classification. Signal Processing, 2013. 93(6): p. 1597-1607.

5. Chen, D., et al., Residual enhanced visual vector as a compact signature for mobile visual search. Signal Processing, 2013. 93(8): p. 2316-2327.

6. Bravo-Solorio, S. and A.K. Nandi, Automated detection and localisation of duplicated regions affected by reflection, rotation and scaling in image forensics. Signal Processing, 2011. 91(8): p. 1759-1770.

7. Zhu, G., et al., SIFT on manifold: An intrinsic description. Neurocomputing, 2013. 113(0): p. 227-233. 